\section{Spironolacton ohne Wirkung bei Herzinfarkt ohne Herzinsuffizienz - aber nicht auf ganzer Linie}

\author{
In der Akuttherapie von Herzinfarktpatienten ohne Herzinsuffizienz blieb Spi- \\ ronolacton in der ALBATROSS-Studie ohne Wirkung. Eine Hoffnung bleibt je- \\ doch: In der Subgruppe der STEMI-Patienten deutete sich ein deutlicher \\ prognostischer Vorteil an.
}

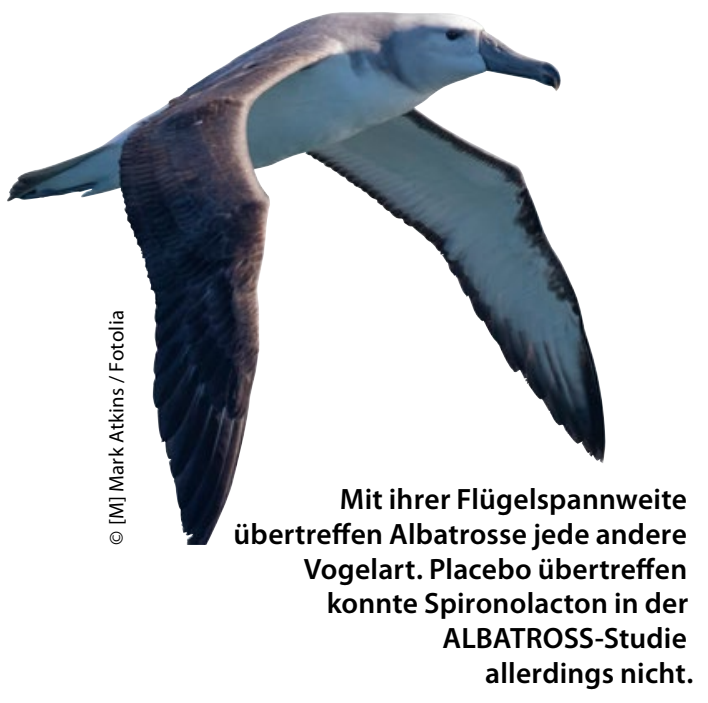

W enn Patienten mit akutem Herzinfarkt einen hohen Aldosteronwert aufweisen, ist dies eindeutig ein schlechtes prognostisches Zeichen. Eine Therapie mit dem Aldosteronblocker Eplerenon verbessert die Prognose von Herzinfarktpatienten, sofern der Patient in den Tagen nach dem Infarkt eine Herzinsuffizienz mit einer Ejektionsfraktion unter $40 \%$ entwickelt.

Ist die Behandlung mit einem Mineralkortikoid-Rezeptor-Antagonisten eventuell auch schon in der Akuttherapie des akuten Herzinfarktes vorteilhaft, ehe sich überhaupt eine Herzschwäche ausprägt? Dieser Frage ist man in der ALBATROSS-Studie ("Aldosterone Lethal effects Blockade in Acute myocardial infarction Treated with or without Reperfusion to improve Outcome and Survival at Six months follow-up“) nachgegangen, deren Ergebnisse der Kardiologe Prof. Gilles Montalescot, Centre Hospitalier Universitaire Pitié-Salpetrière in Paris, auf dem ESC vorstellte.

1.600 Herzinfarktpatienten wurden unselektiert so früh wie möglich - mitunter schon im Krankenwagen - auf eine Therapie mit $200 \mathrm{mg}$ Kaliumcanreonat i. v. und im Anschluss $25 \mathrm{mg} / \mathrm{d}$ Spironolacton für sechs Monate bzw. Placebo randomisiert. Patienten mit unkontrollierten Kalium- oder Kreatininwerten oder anamnestischer Herzinsuffizienz wurden ausgeschlossen. Die Studienteilnehmer waren im Schnitt 58 Jahre alt und wiesen zu ca. $80 \%$ einen STEMI auf, der mit einer PCI behandelt wurde.

\section{Primärer Endpunkt neutral}

Primärer Endpunkt war die Kombination Tod, Wiederbelebung nach Herzstillstand, Kammerflimmern oder Kammertachykardie, ICD-Implantation oder Herzinsuffizienz-Entwicklung. Auf ihn habe die Therapie keinen Einfluss gehabt (11,8 vs. $12,2 \%)$, so Montalescot. $3 \%$ der Patienten entwickelten eine Hyperkaliämie - weniger als erwartet.

\section{Deutliches prognostisches Signal bei STEMI-Patienten}

Hoffnung macht ein Blick in die Subgruppen. Betrachtet man nur die STEMI-Patienten - immerhin das Gros der Patienten - und den härtesten aller Endpunkte - die Gesamtmortalität - so offenbarte sich hier ein erstaunlicher Effekt: Spironolacton reduzierte das Sterberisiko um $80 \%$. Gleichsam erhöhte die Behandlung das Sterberisiko bei den NSTEMI-Patienten auf das 3,5-Fache.

Dieser Befund müsse, so Montalescot, mit großer Vorsicht betrachtet und idealerweise in einer weiteren Studien mit ausschließlich STEMI-Patienten überprüft werden. Sein Fazit: Trotz hoffnungsvoller präklinischer und Registerdaten enttäuscht Spironolacton in der akuten Herzinfarkttherapie, erweist sich aber als sicher. Derzeit gibt es keinen Grund, Aldosteronblocker beim akuten Myokardinfarkt ohne Herzinsuffizienz einzusetzen.

Dr. med. Dirk Einecke

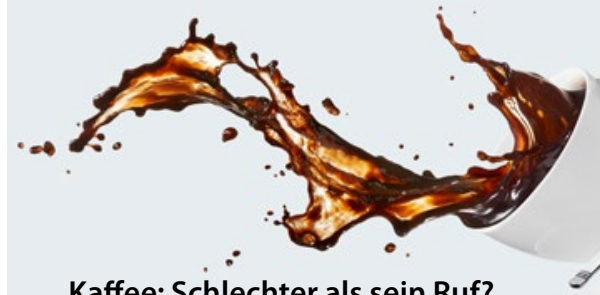

Kaffee: Schlechter als sein Ruf?

Kaffee gilt gemeinhin als Genussmittel, mit dem man seiner Gesundheit noch am ehesten einen Gefallen tut. Umso mehr erstaunt eine Kohortenstudie aus Italien: Junge Hypertoniker, die viel Kaffee trinken, sollen eine höhere kardiovaskuläre Ereignisrate haben. Was ist da los?

Kardiologen um Lucio Mos von der Klinik San Daniele in Udine werteten Daten von 1.201 jungen Erwachsenen mit leichter Hypertonie aus. Dabei fanden sie einen linearen Zusammenhang zwischen Kaffeekonsum und der Quote der Patienten mit behandlungsbedürftiger Hypertonie. Die abstinenten Patienten $(26,3 \%$ der Teilnehmer) wurden zu knapp $60 \%$ behandlungsbedürftig, jene mit starkem Kaffeekonsum (9,9\%) zu über $70 \%$. Das war nach Adjustierung für Alter und Geschlecht mit einer Hazard Ratio von 1,5 statistisch signifikant $(p=0,004)$. Die Italiener berechneten zudem das Risiko kardiovaskulärer Ereignisse, wobei nur 60 Ereignisse in 12,5 Jahren auftraten. Bei moderatem Kaffeegenuss war das Risiko dreifach und bei starkem Genuss vierfach erhöht. Wurden Hypertonie und Prädiabetes einbezogen, schmolz die Signifikanz dahin. Dies deute darauf hin, dass der Kaffeekonsum über den Umweg einer Blutdruckerhöhung bzw. der Begünstigung einer diabetischen Stoffwechsellage zum erhöhten kardiovaskulären Risiko führe, so Mos.

In London wurden die Daten kontrovers diskutiert, da sie im Widerspruch zu vielen anderen Studien stehen. So war der Kaffeekonsum in einer Metanalyse dosisabhängig mit einer niedrigeren Gesamtund kardiovaskulären Mortalität assoziiert. Die meisten Kaffeedaten beziehen sich jedoch auf gesunde Erwachsene, während hier nur junge Patienten mit leichter Hypertonie teilnahmen.

José González Juanatey, Präsident der Spanischen Gesellschaft für Kardiologie, wies daraufhin, dass eine arterielle $\mathrm{Hy}$ pertonie bei jungen Menschen stark von einem überaktiven Sympathikus getrieben werde. Es sei denkbar, dass Koffein in dieser Konstellation den Sympathikus weiter antreibe und so den Blutdruck erhöhe. Bewiesen ist das freilich nicht. Und so wurde dann Mos' Empfehlung, jungen Hypertonikern von starkem Kaffeekonsum abzuraten, vom Podium angesichts derzeit fehlender randomisierter Daten eher kritisch gesehen.

(gvg) 